\title{
Type 2 Diabetes Mellitus Management: A Retrospective Study in Rural General Practice
}

\author{
Ruby Wyett ${ }^{1}$, Blake Peck ${ }^{2}$, Daniel Terry ${ }^{2, *}$ \\ ${ }^{1}$ Ballarat Health Service, Ballarat, Australia \\ ${ }^{2}$ School of Nursing and Healthcare Professions, Federation University Australia, Victoria, Australia
}

Copyright $\odot 2019$ by authors, all rights reserved. Authors agree that this article remains permanently open access under the terms of the Creative Commons Attribution License 4.0 International License

\begin{abstract}
Type 2 Diabetes Mellitus (T2DM) is a chronic, progressive metabolic disease that is an international epidemic. General Practitioners (GPs) are the cornerstones of T2DM management. The aim of this study was to determine the scope of care and management of patients with T2DM within General Practice, while highlighting domains of success and areas where improvement can be made. Demographic and laboratory cross sectional data were collected by examining electronic patient records at one rural General Practice to address the aims of the study. Data included key management parameters of Hemoglobin Alc (HbAlc), estimated Glomerular Filtration Rate (eGFR), microalbuminuria, blood pressure and cholesterol levels, in addition to age, sex, and residential postcode. Further, data regarding the use of insulin, antihypertensive medications and lipid-lowering medications were collected and analyzed. Descriptive and inferential statistics were used and significance was determined at $\mathrm{p} \leq 0.05$. A total of 294 electronic patient records were examined. Glycaemic control was shown to have clinically improved over time, with only $10.8 \%$ of patients having poor control at their latest $\mathrm{HbAlc}$ test. Despite improvement in $\mathrm{HbAlc}$, only $35.4 \%$ of patients had been referred for diabetes education to a diabetes clinic. eGFR showed an overall decline in patient kidney function above the level of decline consistent with aging. Males were significantly more likely to have microalbuminuria with increased severity than females. The study demonstrated an overall clinical improvement in the diabetic control of patients, while identifying key at risk sub-populations. The findings suggest the need for continuous patient orientated management, while highlighting areas for improvement that impact patient health outcomes and avenues for service sustainability greater.
\end{abstract}

Keywords Type 2 Diabetes Mellitus, Diabetes Complications, Family Practice, General Practice, Life Style, Rural Health

\section{Introduction}

Type 2 Diabetes Mellitus (T2DM) is a chronic, progressive metabolic disease that is an international epidemic. As of 2015, one million Australians were affected by T2DM and its prevalence is only expected to rise due to the aging population, the increase in obesity, and sedentary lifestyles [1]. T2DM is predicted to become Australia's leading cause of disease burden, costing over $\$ 14$ billion annually [2]. In addition to the monetary cost, T2DM reduces the life expectancy of those affected by up to 5 years [2].

Australians living in regional, rural and remote regions have drastically higher rates of morbidity and mortality when compared to those living in major cities. Specifically, the National Health Survey reported 6.7\% Australians in outer regional/ remote areas were diagnosed with T2DM, compared to $4.7 \%$ Australians in major cities [3]. Furthermore, people with T2DM who live rurally were found to have more risk factors related to diabetes than their urban counterparts, which is due to the social determinants of health and disparity in health access [4].

In rural settings, GPs play a dominant role in the management of T2DM, with over 2.9 million GP consultations per year related to diabetes in Australia [5]. This increased demand on rural GPs is due to the limited access to specialist services, allied health professionals and other treatment facilities. In rural and remote towns, vast gaps exist between patient management and the targets defined in the guidelines for management of T2DM [5]. Due to this variance, it is vital to examine the specific diabetic complications occurring among patients with T2DM in rural general practice, the current scope of care, management and decision making processes occurring among rural GPs [6]. The management of T2DM is ever evolving and requires up-to-date knowledge particularly in rural areas where very little research and literature exists concerning current GP clinical efficacy [6].

This study was conducted to examine the current efficacy of rural GP T2DM management in relation to 
national guidelines, and identify areas of success and areas for improvement. It was hypothesised that GP management of patients with T2DM, in a regional centre, was in line with the measurable domains of the current guidelines and that the areas of difficulty that GPs highlight would be those notoriously hard to control, such as body mass index (BMI) and waist circumference would be common.

\section{Materials and Methods}

\subsection{Participants}

The patient sample was generated by identifying all active patients who attended a regional General Practice with a diagnosis of T2DM. This resulted in a sample size of 294 patients aged 18 years and older. This study received ethical approval to be conducted.

\subsection{Data Collection}

Demographic data such as age, sex and postcode were collected from electronic patient records retrospectively. Postcodes were used to identify proximity to the clinic, labelling patients as either 'in-town' or 'out-of-town'. Laboratory data including Hemoglobin A1c (HbAlc), estimated Glomerular Filtration Rate (eGFR) and urine microalbumin were collected from the patient's baseline test result and their most recent available test result. Measurements of Blood Pressure, Body Mass Index (BMI) and waist circumference were also collected from baseline and last available measurements. Data on cardiovascular risk was assessed through anti-hypertensive medication use, lipid-lowering medication use, and cardiovascular co-morbidities which included ischemic heart disease (IHD), cerebral vascular diseases (CVD), heart failure (HF), peripheral vascular disease (PVD), aortic aneurism, Acute Myocardial Infarction (AMI) and hypertension. Data regarding insulin use was collected to assess patients with oral medication resistant T2DM. The numbers of referrals to diabetic and renal clinics, both rural and metropolitan, were also identified.

\subsection{Data Analysis}

Data were cleaned, cross-checked and analyzed using Microsoft Excel and SPSS v22. Descriptive statistics were generated from demographic and clinical data and were used to compare and describe various clinical findings, while inferential statistics, such as paired sample t-tests examined group comparisons. Results are shown as means and percentages and the level of significance was determined by two-tailed $\mathrm{p} \leq 0.05$.

\section{Results}

The characteristics of the 294 patients identified with
T2DM indicated that they were predominantly male $(55.4 \%)$, and were over the age of 65 years. The mean age of female patients (66.3 years, 95\%CI: 60.5-72.1) was slightly higher than males (65.2 years, 95\%CI: 60.1-70.3). The majority of patients lived in town, with approximately a fifth of patients living in what was considered 'out-of-town'. Lastly, 14 patients were aged care residents. (Table 1)

Table 1. Demographics of patients with T2DM

\begin{tabular}{|c|c|}
\hline & Frequency n $(\%)$ \\
\hline \multicolumn{2}{|l|}{ Sex } \\
\hline Males & $163(55.4 \%)$ \\
\hline Females & $131(44.6 \%)$ \\
\hline \multicolumn{2}{|l|}{ Age } \\
\hline $18-55$ years & $57(19.4 \%)$ \\
\hline $56-65$ years & $86(29.3 \%)$ \\
\hline $66-75$ years & $82(27.8 \%)$ \\
\hline $76+$ years & $69(23.5 \%)$ \\
\hline \multicolumn{2}{|l|}{ Residence } \\
\hline 'In-town' residents & $233(79.3 \%)$ \\
\hline 'Out-of-town' residents & $61(20.7 \%)$ \\
\hline \multicolumn{2}{|l|}{ Attends Diabetes Clinic } \\
\hline Rural & $89(30.3 \%)$ \\
\hline Metro & $7(2.4 \%)$ \\
\hline Both & $8(2.7 \%)$ \\
\hline \multicolumn{2}{|l|}{ Attends Renal Clinic } \\
\hline Rural & $21(7.1 \%)$ \\
\hline Metro & $3(1.0 \%)$ \\
\hline Both & $0(0.0 \%)$ \\
\hline Lives in Aged Care Facility & $14(4.7 \%)$ \\
\hline \multicolumn{2}{|l|}{ Diabetes Care plan } \\
\hline Within 12 months & $227(77.2 \%)$ \\
\hline Longer than 12 months & $29(9.8 \%)$ \\
\hline Does not have care plan & $38(13.0 \%)$ \\
\hline
\end{tabular}

\subsection{Glycaemic Control}

Patient baseline glycaemic control measures indicated that $77.6 \%$ had adequate to very good control (Table 2). The mean time between baseline and most recent glycaemic control measures was 2.9 years, with control improving clinically among $79.8 \%$ of patients. Despite the clinical improvement, the mean difference of $\mathrm{HbAlc}$ levels over time was only $1.01 \mathrm{mmol} / \mathrm{mol}(95 \% \mathrm{CI}:-0.61-$ 2.64) and not statistically significant, $\mathrm{t}(276)=1.230$, $\mathrm{p}=.220$.

Over time, measures of glycaemic control varied little between sexes and age groups. Non-smokers had slightly more success in clinically improving their glycaemic control than active or ex-smokers with $4.7 \%$ less patients experiencing poor glycaemic control in the non-smoking group in their most recent measures. The mean difference of $\mathrm{HbAlc}$ levels between non-smokers and active or ex-smokers was $2.54 \mathrm{mmol} / \mathrm{mol}(95 \% \mathrm{CI}$ : 0.45-4.62) and was demonstrated to be statically significant, $\mathrm{t}(120)=$ $2.415, \mathrm{p}=.017$. Among the 294 patients, only 104 (35.4\%) had been referred to and attended an external diabetes clinic for T2DM education and patient driven management strategies. 
Table 2. Glycaemic control among patients with T2DM over time

\begin{tabular}{ccc}
\hline & Initial Test $\mathrm{n}(\%)$ & Most recent Test $\mathrm{n}(\%)$ \\
\hline HbA1c (mmol/mol) & & $162(58.5 \%)$ \\
Very Good Control (45-53) & $169(61.0 \%)$ & $59(21.3 \%)$ \\
Adequate control (54-64) & $46(16.6 \%)$ & $26(9.4 \%)$ \\
Suboptimal Control (65-75) & $21(7.6 \%)$ & $47(10.8 \%)$ \\
Poor control $(>75)$ & $58(14.8 \%)$ & \\
\hline
\end{tabular}

Table 3. Kidney function monitoring of patients with T2DM over time

\begin{tabular}{ccc}
\hline & Initial Test & Most recent Test \\
\hline eGFR $(\mathrm{mL} / \mathrm{min} / 1.73 \mathrm{~m} 2)$ & & $88(32.1 \%)$ \\
Stage 1 $(>90)$ & $96(35.1 \%)$ & $114(41.6 \%)$ \\
Stage 2 (60-89) & $133(48.5 \%)$ & $40(14.6 \%)$ \\
Stage 3a (45-59) & $30(10.9 \%)$ & $17(6.2 \%)$ \\
Stage 3b (30-44) & $9(3.3 \%)$ & $14(5.1 \%)$ \\
Stage 4 (15-29) & $6(2.2 \%)$ & $1(0.4 \%)$ \\
Stage 5 (>15) & $0(0.0 \%)$ & $1(0.6 \%)$ \\
\hline Urine Microalbumin (mg/mmol) & & $104(68.9 \%)$ \\
Men & $5(30.5 \%)$ \\
Microalbuminuria (2.5-25) & $98(64.9 \%)$ & $46(30.5 \%)$ \\
Macroalbuminuria $(>25)$ & $48(31.8 \%)$ & $43(36.4 \%)$ \\
Women & & $51(43.2 \%)$ \\
Normal $(>3.5)$ & $45(38.1 \%)$ & $24(20.3 \%)$ \\
Microalbuminuria $(3.5-35)$ & $48(40.7 \%)$ &
\end{tabular}

\subsection{Diabetic Sequelae Screening}

\subsubsection{Microvascular Complications}

Patient eGFR and urine microalbumin were examined in order to assess kidney function and the presence of diabetic nephropathy. At baseline, $83.6 \%$ of patients had an eGFR of above $60 \mathrm{~mL} / \mathrm{min} / 1.73 \mathrm{~m} 2$ (Kidney function stages 1 and 2 - adequate renal function). The remaining $16.4 \%$ had kidney function below this level and 6 of these patients had eGFRs of $15-29 \mathrm{~mL} / \mathrm{min} / 1.73 \mathrm{~m} 2$ (Kidney function stage 4) (Table 3).

Over time (mean 3.2 years), the number of patients with stage 1 and 2 kidney function had decreased by almost $10.0 \%$ while the number of patients with stage 4 had increased to 14 . This decline in renal function over time lead to a mean eGFR difference of $3.10 \mathrm{~mL} / \mathrm{min}$ per 1.73 $\mathrm{m} 2$ (95\%CI: 1.58-4.62) and was indicated to be statistically significant $\mathrm{t}(273)=4.018, \mathrm{p}=.001$. The rate of eGFR declined among patients over time was 1.06 $\mathrm{mL} / \mathrm{min} / 1.73 \mathrm{~m} 2$ per year, which is above the normal age related decline rate of $0.8 \mathrm{~mL} / \mathrm{min} / 1.73 \mathrm{~m} 2$ per year.

The examination of urine microalbumin levels supported the overall decrease in patient kidney function. At baseline, $22.6 \%$ were excreting acceptable levels of albumin, and the vast majorities $(90 \%)$ were females. When comparing baseline microalbumin results between sexes, $34.8 \%$ more men than women were excreting either micro or macro levels of albumin. This difference remained consistent over time (mean 2.7 years), with $35.8 \%$ more men than women exhibiting abnormal levels of albuminuria. This increase indicated that women's albuminuria increased an average of $12.5 \mathrm{mg} / \mathrm{mol}(95 \% \mathrm{CI}$ :
11.29-13.70), while the men's increased by an average of $65.8 \mathrm{mg} / \mathrm{mol}$ (95\%CI: 60.43-71.16). These microalbumin differences were statistically significant $\mathrm{t}(152)=2.897$ $\mathrm{p}=.004, \mathrm{t}(120)=2.780, \mathrm{p}=.005$, among men and women respectively.

\subsubsection{Macrovascular Complications.}

The screening of macrovascular complications was assessed. Sixty-seven percent of patients had initially higher than normal levels of cholesterol, $51.8 \%$ male and $55.3 \%$ aged under 65 years. Over a mean of 3.2 years higher than normal levels of cholesterol decreased by $8.8 \%$ or a mean of $0.28 \mathrm{mmol} / \mathrm{L}(95 \% \mathrm{CI}: 0.15-0.41)$, a statistically significant decrease $\mathrm{t}(274)=4.278, \mathrm{p}=.001$ (Table 4). Systolic blood pressure was found to be more tightly controlled, with $43.4 \%$ of patients at the target of below $130 \mathrm{mmHg}$ at their first measurement. This improved over time, with $50.3 \%$ reaching a mean difference of $12.24 \mathrm{mmHg} \quad(95 \% \mathrm{CI}$ : 9.55-14.93), clinically and statistically significant, $\mathrm{t}(197)=8.974$, $\mathrm{p}=.001$.

Body Mass Index and waist circumference showed $93.6 \%$ of patients were initially overweight or obese (Table 4). The most recent measurement indicated those overweight or obese had decreased by $4.9 \%$, a mean of $0.38 \mathrm{Kg} / \mathrm{M} 2$ (95\%CI: $-0.16-0.78)$, and not statistically significant $\mathrm{t}(275)=0.187, \mathrm{p}=.852$. The most common macrovascular complications were ischaemic heart disease $(22.4 \%)$ and cerebrovascular disease $(10.2 \%)$, while diagnosis of acute myocardial infarction, heart failure and peripheral vascular disease were low within the cohort $(6.8 \%, 6.5 \%$ and $2.4 \%$ respectively). 
Table 4. Cardiovascular health monitoring of patients with T2DM over time

\begin{tabular}{|c|c|c|}
\hline & Initial Test & Most recent Test \\
\hline \multicolumn{3}{|l|}{ Total Cholesterol $(\mathrm{mmol} / \mathrm{L})$} \\
\hline$\leq 4$ & $97(33.0 \%)$ & $123(41.8 \%)$ \\
\hline$>4$ & $197(67.0 \%)$ & $171(58.2 \%)$ \\
\hline \multicolumn{3}{|c|}{ Systolic Blood Pressure (mmHg) } \\
\hline$\leq 130$ & $126(43.4 \%)$ & $146(50.3 \%)$ \\
\hline $131-140$ & $60(20.7 \%)$ & $57(19.7 \%)$ \\
\hline $141-150$ & $41(14.1 \%)$ & $44(15.2 \%)$ \\
\hline$\geq 151$ & $63(21.7 \%)$ & $43(14.8 \%)$ \\
\hline \multicolumn{3}{|l|}{ BMI $\left(\mathrm{kg} / \mathrm{m}^{2}\right)$} \\
\hline Underweight $(<18.5)$ & $0(0.0 \%)$ & $0(0.0 \%)$ \\
\hline Normal weight (18.5-24.9) & $18(6.4 \%)$ & $32(11.3 \%)$ \\
\hline Overweight (25-29.9) & $89(31.4 \%)$ & $82(29.0 \%)$ \\
\hline Obesity I (30-34.9) & $101(35.7 \%)$ & $92(32.5 \%)$ \\
\hline Obesity II (35-39.9) & $43(15.2 \%)$ & $49(17.3 \%)$ \\
\hline Obesity III $(\geq 40)$ & $32(11.3 \%)$ & $28(9.9 \%)$ \\
\hline \multicolumn{3}{|l|}{ Waist Circumference (cm) } \\
\hline \multicolumn{3}{|l|}{ Men } \\
\hline Good $(<94)$ & $7(4.5 \%)$ & $9(5.8 \%)$ \\
\hline Increased risk (94-101.9) & $31(19.9 \%)$ & $30(19.2 \%)$ \\
\hline High risk $(\geq 102)$ & $118(75.6 \%)$ & $117(75.0 \%)$ \\
\hline \multicolumn{3}{|l|}{ Women } \\
\hline Good $(<80)$ & $3(2.5 \%)$ & $3(2.5 \%)$ \\
\hline Increased risk (80-87.9) & $6(5.0 \%)$ & $7(5.8 \%)$ \\
\hline High risk $(\geq 88)$ & $111(92.5 \%)$ & $110(91.7 \%)$ \\
\hline
\end{tabular}

\subsubsection{Medications}

The number of patients on common medications for the treatment of diabetic sequelae (such as ACEi, ARBs, Statins and other lipid lowering medications) as well as insulin were studied (see Figure 1). The findings demonstrated that despite the availability of prescription medication, approximately $50 \%$ of patients were still above the recommended physiological parameters for the corresponding measurable outcomes.

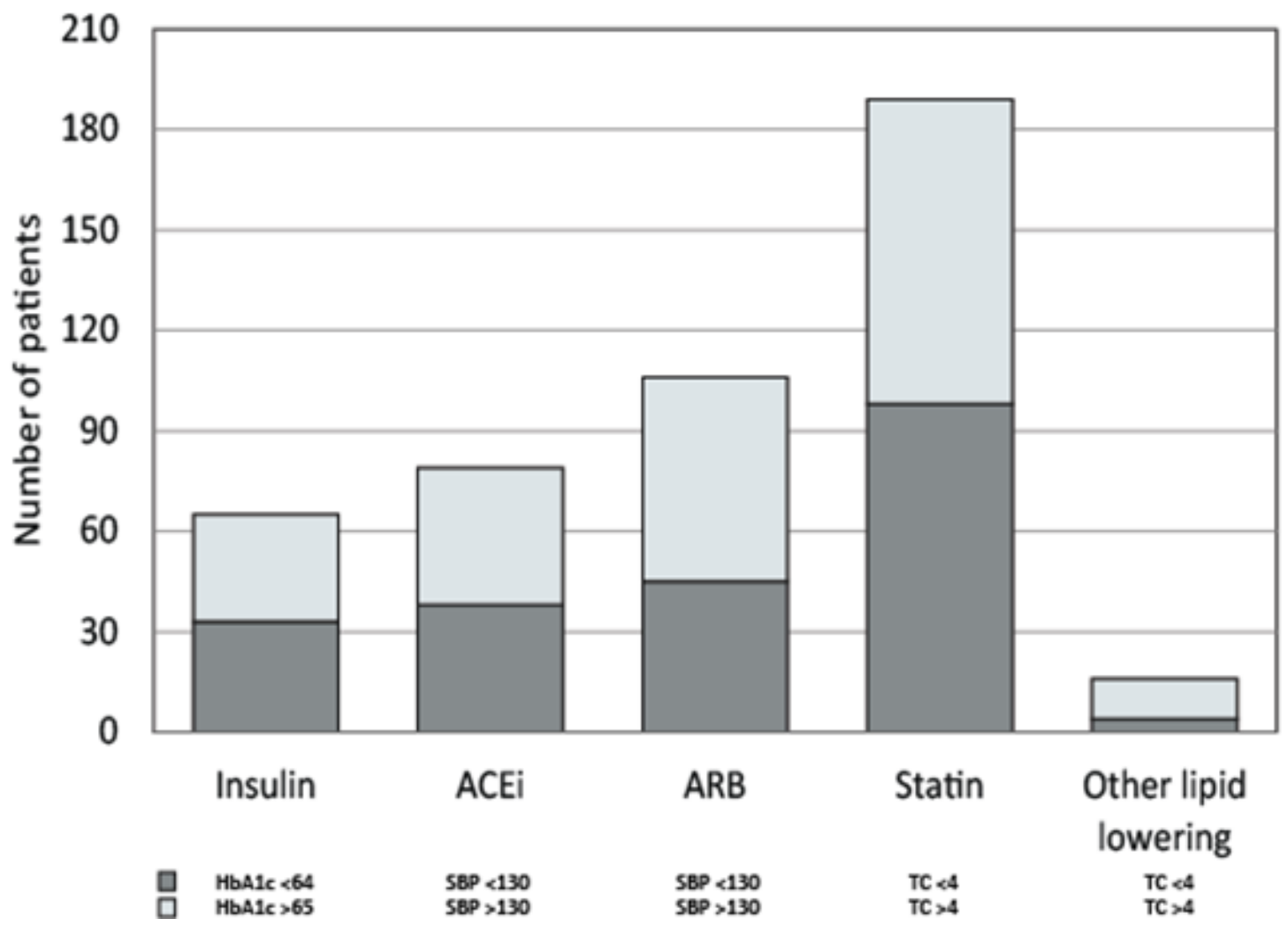

Note: SBP=Systolic Blood Pressure, TC=Total Cholesterol

Figure1. Frequency of common medications and their clinical outcome 


\subsubsection{Variance between 'In-town' and 'Out-of-town'}

Of the 294 patients, $79.3 \%$ lived 'in-town'. Glycaemic control measures between cohorts varied little, with a slightly higher percentage of 'out-of-town' patients $(81.5 \%)$ achieved adequate to very good control compared to 'in-town' patients (79.3\%). However, the 'out-of-town' cohort also had a slightly higher percentage with poor diabetes control than observed elsewhere (13\% and $10.3 \%$ respectively). This did not correlate with the difference in kidney function where the 'out-of-town' cohort had a much higher percentage with good kidney function $(90.7 \%$ versus $69.5 \%$ with eGFR $>60 \mathrm{~mL} / \mathrm{min} / 1.73 \mathrm{~m} 2)$. There was little to no variance among other domains when comparing 'in-town' with 'out-of-town' cohorts. However, BMI and total cholesterol were the exception, where the 'out-of-town' cohort was slightly more overweight with a higher percentage of patients with above normal cholesterol.

\section{Discussion}

Type 2 Diabetes Mellitus is a complex, chronic illness requiring continuous medical care with multifactorial risk-reduction strategies beyond simply glycaemic control [7]. If undiagnosed or poorly managed, T2DM has a devastating systemic impact and numerous sequelae including coronary artery disease, stroke, nephropathy, neuropathy and retinopathy [8]. Therefore, timely diagnosis and optimal management of T2DM is critical to reducing the long term impact and risk of diabetic complications. The aim of this retrospective study was to assess the current efficacy of T2DM management in the rural GP setting in relation to the national guidelines.

The management of T2DM was found to be compliant with the National Diabetes Australia guidelines in most domains and highlighted some areas for improvement. As such, it was indicated that $77.6 \%$ of patients had adequate to very good glycaemic control at baseline and $79.8 \%$ of patients at their most recent visit were achieving the recommended targets for glycaemic control. When compared to a number of earlier international studies, very good control $(\mathrm{HbAlc} \leq 53 \mathrm{mmol} / \mathrm{mol})$ was described in these primary health care sites as ranging from $21 \%$ to $52 \%$ among patients.[7] However, primary health care studies from Spain, Oman, Singapore and Australia, where patient numbers ranged from between 445 to 3893 , had HbA1c levels that ranged between $32 \%-47 \%$ on the very good control measure and were therefore in contrast to findings from this current study [8-11]. This suggests that the overall and multifaceted management of glycaemic control by those GPs whose patient data is represented here have been highly-effective in the management of T2DM.

In addition to $\mathrm{Hb} 1 \mathrm{Ac}$, both eGFR and microalbumin levels indicated that overall kidney function declined over time with very little differences being observed between these measures, however eGFR levels decreased beyond the current 'expected' normal rates of decline.[12, 13] In a number of studies, eGFR levels among patients with diabetes remained a concern particularly at the primary care level.[14] Although the bulk of patients with early stage chronic kidney disease are often most appropriately managed in the primary care setting [15], the greater than normal age related decline identified within this study would suggest that a need for greater emphasis on kidney function management at the primary care level. The challenge for primary care practice however, is that other studies have shown that when incorporating strategies to address eGFR decline by a multidisciplinary care teams patients have better outcomes when compared to those who receive standard care from their GP.[16, 17]

The analysis revealed diabetic management medications had poor clinical efficacy in achieving the recommended outcomes among patients, with less than $50 \%$ of patients achieving clinical targets. This may suggest issues of patient non-compliance to both diabetic education and medication management, and a broader concern of patients regarding medication side effects, such as those commonly experienced mongst those prescribed lipid-lowering medications.[18] This highlights the need for GPs to further examine medication education, medication efficacy vs medication side effects which informs patient compliance, patient preference, while balancing the less tight control seen among the elderly, and the need to increase medication dosage, or to augment therapy with additional medications. Further, and interestingly, it has been shown elsewhere that many of the challenges observed in the primary care of people with diabetes may also be overcome through improved working alliances between GPs and patient, where primary care providers with greater levels of empathy have been shown to have higher proportions of adequate $\mathrm{HbA1c}$ control and lower levels of cholesterol amongst their patient group. [19]

Following on from this, other models of care, such as shared care, have been developed for the care of patients with T2DM.[20, 21, 22] Some have had positive, yet non-significant $\mathrm{HbAlc}$ control, while others have had positive outcomes for blood pressure and chronic kidney disease,[23] with the majority of outcomes being mixed.[22, 24, 25] Overall, it has been argued that supportive services have had a positive impact on GP management of patients and patient diabetic awareness, motivation and behaviours.[21]

This retrospective study has highlighted that very few clinical comparisons reached statistical significance, however, reflects other similar studies,[21, 22, 24, 25] Despite this, the study does highlight that GPs who undertake the bulk of patient care in rural and regional areas with limited access to peripheral support services, still lead to the positive clinical management of their 
patients. Notwithstanding these positive findings, there is sufficient evidence within the literature to suggest that where primary healthcare providers are supported by multidisciplinary health teams, we see the most beneficial outcomes for patients in geographically isolated regions. [19]

\subsection{Study Limitations}

Electronic patient files did not always contain both a baseline and the latest test result for the laboratory data or other measures completed during the consultations. This may be due to a number of reasons which include, the patient not being tested, being a new patient at the clinic, or the patient attending various clinics within the town. Further, it was suggested that GPs may not have always entered the result of allied health visits, such as optical assessments, into the electronic patient files. Demographic data collection was limited with large amounts of data on ethnicity not being collected and thus, any correlation with diabetic glycaemic control could not be analysed.

\section{Conclusions}

Considering the rising rate of T2DM, especially in rural areas, it is important to examine the effectiveness of clinical strategies of management and compliance amongst health professionals with the most contemporary guidelines of management. As General Practice is the cornerstone of T2DM management, particularly in rural areas where the peripheral support services are scarce, it is imperative that GPs are able to access regionally specific data that examines the effectiveness of patient care on tangible data such as glycaemic control measures and associated micro and macro vascular risk factors.. Overall, this study provides vital information regarding the management of T2DM in the rural GP setting. It highlights that diabetes management is adequately maintained within the General Practice settings examined through this work, and suggests a positive trend towards overall improvement in glycaemic control for the patients engaged with General Practice. One clear example of this was patients with poor kidney function who were identified as being adequately managed in General Practice with referral to renal clinics as appropriate. While overall positive, this work importantly identifies those areas that are open to improvement. For example, some differences between 'in-town' and 'out-of-town' patients, among prescribed medication and their clinical outcomes and referrals to diabetic clinics, identified opportunities for greater focus when patients regularly seek care. The findings from this study are useful for not only the General Practice where the study was conducted, but also other regional Practices who are seeking evidence of their impact on the communities they serve.

\section{Acknowledgements}

This research has been supported by the Australian Government through the School of Nursing and Healthcare professions at Federation University Australia. Initial research was supported by the Australian Government Department of Health through the Rural Health Multidisciplinary Training Program.

\section{REFERENCES}

[1] Furler J, Hii JWS, Liew D, Blackberry I, Best J, Segal L, et al. The "cost" of treating to target: cross-sectional analysis of patients with poorly controlled type 2 diabetes in Australian general practice. BMC Family Practice. 2013; 14.

[2] Furler JS, Young D, Best J, Patterson E, O'Neal D, Liew D, et al. Can primary care team-based transition to insulin improve outcomes in adults with type 2 diabetes: the stepping up to insulin cluster randomized controlled trial protocol. Implement Sci. 2014; 9: 20-8.

[3] Australian Bureau of Statistics. Australian Health Survey: First Results, 2014-15 2015 [Available from: http://www.abs.gov.au/ausstats/abs@.nsf/Lookup/by\%20S ubject/4364.0.55.001 2014-15 Main\%20Features $\sim$ Diabet es\%20mellitus 12 .

[4] Page-Carruth A, Windsor C, Clark M. Rural self-reliance: the impact on health experiences of people living with type II diabetes in rural Queensland, Australia. Int J Qual Stud Health Well-being. 2014; 9.

[5] Thepwongsa I, Kirby C, Paul C, Piterman L. Management of type 2 diabetes: Australian rural and remote general practitioners' knowledge, attitudes, and practices. Rural Remote Health. 2014; 14:2499-519.

[6] Glenister K, Terry D. Chronic ill health in Hume: A scoping exercise to navigate research. Shepparton: Department of Rural Health, the University of Melbourne 2015.

[7] Wee SL, Tan CG, Ng HS, Su S, Tai VU, Flores JV, et al. Diabetes outcomes in specialist and general practitioner settings in Singapore: challenges of right-siting. Annals Academy of Medicine Singapore. 2008; 37(11):929-35.

[8] Alonso-Fernández M, Mancera-Romero J, Mediavilla-Bravo JJ, Comas-Samper JM, López-Simarro F, Pérez-Unanua MP, et al. Glycemic control and use of Alc in primary care patients with type 2 diabetes mellitus. Primary Care Diabetes. 9(5):385-91.

[9] Al-Lawati JA, Barakat MN, Al-Maskari M, Elsayed MK, Al-Lawati AM, Mohammed AJ. HbA1c Levels among Primary Healthcare Patients with Type 2 Diabetes Mellitus in Oman. Oman Medical Journal. 2012; 27(6):465-70.

[10] Quah JH, Liu Y, Luo N, How C, Tay E. Younger adult type 2 diabetic patients have poorer glycaemic control: a cross-sectional study in a primary care setting in Singapore. BMC Endocrine Disorders. 2013; 13(1):18-28.

[11] MacIsaac RJ, Jerums G, Weekes AJ, Thomas MC. Patterns 
of glycaemic control in Australian primary care (NEFRON 8). Internal medicine journal. 2009; 39(8):512-8.

[12] Phoon R. Chronic kidney disease in the elderly Assessment and management. Australian Family Physician. 2012; 41: 940-4.

[13] Perkins BA, Nelson RG, Ostrander BE, Blouch KL, Krolewski AS, Myers BD, et al. Detection of renal function decline in patients with diabetes and normal or elevated GFR by serial measurements of serum cystatin C concentration: results of a 4-year follow-up study. Journal of the American Society of Nephrology. 2005; 16(5):1404-12.

[14] Ravera M, Noberasco G, Re M, Filippi A, Gallina AM, Weiss U, et al. Chronic kidney disease and cardiovascular risk in hypertensive type 2 diabetics: a primary care perspective. Nephrology Dialysis Transplantation. 2009; 24(5):1528-33.

[15] Sakhuja A, Hyland J, Simon JF. Managing advanced chronic kidney disease: a primary care guide. Cleve Clin J Med. 2014; 81(5):289-99.

[16] Wyatt C, Konduri V, Eng J, Rohatgi R. Reporting of Estimated GFR in the Primary Care Clinic. American Journal of Kidney Diseases. 2007; 49(5):634-41.

[17] Bayliss EA, Bhardwaja B, Ross C, Beck A, Lanese DM. Multidisciplinary Team Care May Slow the Rate of Decline in Renal Function. Clinical Journal of the American Society of Nephrology. 2011; 6(4):704-10.

[18] Rushforth B, McCrorie C, Glidewell L, Midgley E, Foy R. Barriers to effective management of type 2 diabetes in primary care: qualitative systematic review. Br J Gen Pract. 2016; 66(643):e114-e27.

[19] Jones A, Vallis M, Cooke D, Pouwer F. Working together to promote diabetes control: A practical guide for diabetes health care providers in establishing a working alliance to achieve self-management support. Journal of diabetes research. 2016; 2016.

[20] Davidson P, Halcomb E, Hickman L, Phillips J, Graham B. Beyond the rhetoric: what do we mean by a 'model of care'? Australian Journal of Advanced Nursing. 2006; 23(3):47-55.

[21] Swerissen H, Stanton J, Marshall S, Peterson C, Tilgner L. An Evaluation of a Shared Care Diabetes Project. Australian Journal of Primary Health. 2000; 6(2):30-7.

[22] Smith SM, Allwright S, O'Dowd T. Effectiveness of shared care across the interface between primary and specialty care in chronic disease management. Cochrane Database of Systematic Reviews 2007(3).

[23] Scherpbier-de Haan ND, Vervoort GM, van Weel C, Braspenning JC, Mulder J, Wetzels JF, et al. Effect of shared care on blood pressure in patients with chronic kidney disease: a cluster randomised controlled trial. British Journal of General Practice. 2013; 63(617):e798-e806.

[24] van Hateren KJ, Drion I, Kleefstra N, Groenier KH, Houweling ST, van der Meer K, et al. A prospective observational study of quality of diabetes care in a shared care setting: trends and age differences (ZODIAC-19). BMJ open. 2012; 2(4):e001387.
[25] Smith SA, Shah ND, Bryant SC, Christianson TJH, Bjornsen SS, Giesler PD, et al. Chronic Care Model and Shared Care in Diabetes: Randomized Trial of an Electronic Decision Support System. Mayo Clinic Proceedings. 2008; 83(7):747-57. 